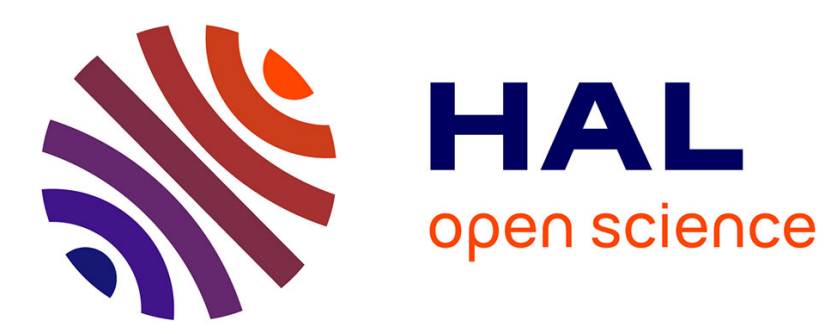

\title{
Highly Anisotropic Rigidity of "Ribbon-like" Polymers: I. Chain Conformation in Dilute Solutions
}

Irina Nyrkova, Alexander Semenov, Jean-François Joanny, Alexei Khokhlov

\section{To cite this version:}

Irina Nyrkova, Alexander Semenov, Jean-François Joanny, Alexei Khokhlov. Highly Anisotropic Rigidity of "Ribbon-like" Polymers: I. Chain Conformation in Dilute Solutions. Journal de Physique II, 1996, 6 (10), pp.1411-1428. 10.1051/jp2:1996139 . jpa-00248378

\section{HAL Id: jpa-00248378 https://hal.science/jpa-00248378}

Submitted on 1 Jan 1996

HAL is a multi-disciplinary open access archive for the deposit and dissemination of scientific research documents, whether they are published or not. The documents may come from teaching and research institutions in France or abroad, or from public or private research centers.
L'archive ouverte pluridisciplinaire HAL, est destinée au dépôt et à la diffusion de documents scientifiques de niveau recherche, publiés ou non, émanant des établissements d'enseignement et de recherche français ou étrangers, des laboratoires publics ou privés. 


\title{
Highly Anisotropic Rigidity of "Ribbon-Like" Polymers: I. Chain Conformation in Dilute Solutions
}

Irina A. Nyrkova $\left({ }^{1,2}\right)$, Alexander N. Semenov $\left({ }^{1,2,3,4}\right)$, Jean-Francois Joanny $\left({ }^{2, *}\right)$ and Alexei R. Khokhlov $\left({ }^{1}\right)$

( ${ }^{1}$ Physics Department, Moscow State University, Moscow 117234, Russia

$\left({ }^{2}\right)$ Institut Charles Sadron, 6 rue Boussingault, 67083 Strasbourg Cedex, France

( $\left.{ }^{3}\right)$ Department of Applied Mathematics, University of Leeds, Leeds LS2 9JT, UK

$\left({ }^{4}\right)$ Nesmeyanov Institute of Organo-Element Compounds of Russian Academy of Science, 28 Vavilova Str., Moscow 117812, Russia

(Received 12 May 1995, revised 25 April 1996, accepted 2 July 1996)

PACS.61.25.Hq - Macromolecular and polymer solutions; polymer melts; swelling

PACS.61.30.-v - Liquid crystals

PACS.64.70.-p - Specific phase transitions

\begin{abstract}
We discuss the solution properties of polymers with a highly anisotropic rigidity which bend rather freely in a plane (the plane of main flexibility) and are extremely rigid in the direction perpendicular to this plane. Examples of these polymers are the ladder polymers recently synthesized or living polymers formed by the aggregation of peptide rodlike fragments. These polymers have a much higher out of plane persistence length $l_{2}$ than their in-plane persistence length $l$. In the first paper of this series, we mostly investigate the conformation of single chains in solution (at extremely low concentrations). The conformation of an isolated chain with a highly anisotropic rigidity essentially depends on the dimensionless parameter $\Omega=l_{2} d^{2} l^{-3}$ where $d$ is the chain diameter. For small values of $\Omega,\left(l_{2} \sim l\right)$, the chain behaves as a standard semiflexible chain with isotropic rigidity. For large values of $\Omega,(\Omega \gtrless 1)$, the chain adopts a one-dimensional rodlike conformation at length scales smaller than $l$, an anisotropic disc-like conformation at intermediate scales (corresponding to a contour length $L$ such that $l \lesssim L \lesssim l_{2}$ ) and a three-dimensional swollen coil conformation at larger length scale. In the intermediate range of $\Omega, l^{2} / d^{2} \lesssim \Omega \lesssim 1$, the same three regimes are expected but the excluded volume interactions do not play any role in the disc-like regime. At the end of the paper we discuss qualitatively the possible liquid crystalline phases (with nematic or smectic symmetry) which can emerge in these solutions at higher concentration.
\end{abstract}

\section{Introduction}

The conformation and the phase behavior of semiflexible polymer chains in solution are now rather well understood [1]. Both the properties of isotropic solutions [2] and the isotropicnematic transition [3-5] have been studied quite thoroughly. The detailed structure of the solution is controlled by the asymmetry ratio $d / l$ ( $d$ is the diameter and $l$ is the Kuhn segment of the chain) and by the total contour length $L$ (or, in another language, by the number

$\left(^{*}\right)$ Author for correspondence (e-mail: joanny@janus.u-strasbg.fr) 

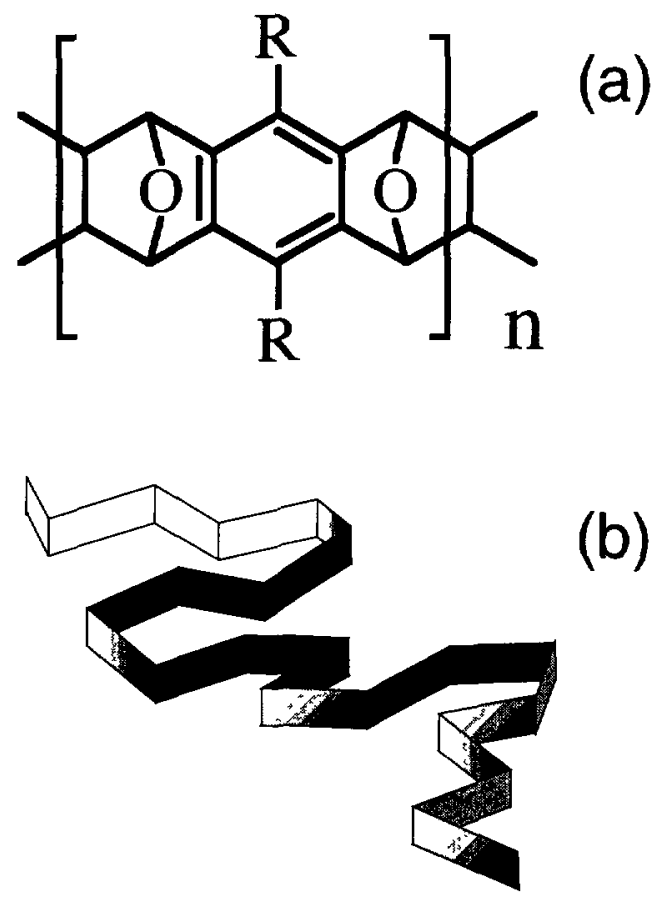

Fig. 1. - (a) Ladder polymer chain with mirror symmetry synthesized in references $[7,8]$; (b) local conformation of the polymer - random walk in the horizontal plane.

of monomeric units, $N$, the excluded volume of the monomer, $B$, and their size, $a$ ). Scaling theories have been successfully constructed, the results can be summarized on diagrams showing the various possible regimes in the variables $\{L, d, l, \Phi\}$ (or $\{N, a, B, c\}$ ), where $c$ is the polymer concentration and $\Phi$ is the corresponding polymer volume fraction $[2,6]$.

However, up to now, only polymers with an isotropic rigidity have been studied theoretically: in all the models, the chains actually have a rotational symmetry around their main axis. The term rotational symmetry includes here not only the pure free-rotation between consecutive monomers, but also all the situations where there are short-range correlations in the rotation angles (e.g. as in trans/gauche models of polyethylene or in the helical structure of DNA). The general configuration of isotropic semiflexible polymer molecules is either "rodlike" for very short chain fragments, $(L \lesssim l$ ) or "coil-like" for very long chains, (for $L \gtrsim l$ ); the chain sizes along the three directions are then equal, $R_{x} \sim R_{y} \sim R_{z}$.

Polymers with a new type of rigidity were recently synthesized and are now available in soluble forms $[7,8]$. They belong to the class of so-called ladder or ribbon polymers with a structure based on two independent, inter-tied strands of bonds. The monomers which form ladder polymers often have mirror symmetry (see Fig. 1a), and hence the corresponding polymers have the shape of two-dimensional coils. They rather freely bend inside one (say the horizontal) plane and are completely rigid in the perpendicular direction (see Fig. 1b). The flexibility inside the horizontal plane is due to endo-exo isomer transitions, so that the polymer shows random walk statistics in this plane. Possible deviations from the horizontal plane are due to a bending or a twisting of the double stranded chain, which require a much larger energy. The thermal fluctuations perturb the pure two-dimensional structure of the chain, and 
at large enough length scales it forms a disc-like object. At even larger length scales the out of plane fluctuations become dominant and a $3 \mathrm{~d}$ coil shape is expected. As the concentration of the solution is increased, the anisotropic configuration of the chain leads to the formation of liquid crystalline ordered phases with a variety of different symmetries.

This paper starts a series of publications devoted to polymers with a highly anisotropic rigidity. Our aim is to construct a scaling theory for the polymer structure in solution. We discuss both the chain conformation in dilute solutions and the liquid crystalline organization in more concentrated solutions. This first paper deals mainly with the single chain conformation (dilute solutions).

The paper is organized as follows. In the next section, we formulate our model for the description of ribbon-like polymers (or, more generally polymers with two-dimensional flexibility, or with planar stiffness). In Section 3 we classify the various possible conformations (shapes) of isolated chains with planar rigidity and clarify the role of the rigidity anisotropy parameter $\Omega$. In the limit where $\Omega \gtrsim 1$, it is possible to formulate a scaling theory for the structure of ribbon chains; this is presented in subsection 3.3. In Section 4 we briefly discuss the various possible liquid crystalline phases which could appear in more concentrated solutions. Finally the last section is devoted to a discussion of our results and to some concluding remarks.

\section{Polymer Chains with Anisotropic Rigidity}

The rigidity of ordinary semiflexible polymers is often characterized by the Kuhn segment, $l$. For an ideal chain of contour length $L$, the mean square end-to-end distance $\left\langle R^{2}\right\rangle$ is proportional to $L$, and by definition $[9,11,24]$

$$
\left\langle R^{2}\right\rangle=L l
$$

for $L \gg l$. Although the ground state of the chain (obtained when the temperatures $T \rightarrow 0$ ) is rod-like, the thermal fluctuations at finite $T$ always make the chain isotropic on large length scales. One can then introduce the so-called persistence length, $l_{\mathrm{p}}[1,9-11]$, which characterizes the orientation memory of the vector $\mathbf{t}(s)$ tangent to the chain backbone:

$$
\langle\mathbf{t}(0) \mathbf{t}(s)\rangle=\exp \left(-s / l_{\mathrm{p}}\right)
$$

where $s$ is the distance along the chain contour. Generally $l_{\mathrm{p}} \sim l$, and hence in the scaling theories, only one parameter $(l)$ is necessary $[1,9]$.

We now describe the structure of a chain with planar stiffness sketched in Figure 1b. The chain has a finite stiffness both in "the plane of main flexibility" (PMF) (the horizontal plane in Fig. 1b) and in the perpendicular direction (outside this horizontal plane), but the out of plane rigidity is higher. We thus need two rigidity parameters to characterize the chain statistics: $l$ characterizes the rigidity of the chain inside the "plane of main flexibility", and $l_{2}$ the "out-of-plane" rigidity

$$
l \lesssim l_{2}
$$

In the absence of excluded volume interactions (this ideal situation however never realizes for a real two-dimensional polymer $[1,6])$ the mean-square end-to-end distance is governed by the Gaussian law (2.1).

The second rigidity, $l_{2}$, is in fact the persistence length for the PMF. If $l_{2} \rightarrow \infty$ the trajectory of the chain is completely flat and the corresponding plane can be characterized by its normal vector, $\mathbf{n}$. If $l_{2} \gg l$, we can divide the chain into subunits of contour length $\lambda\left(l \ll \lambda \ll l_{2}\right)$ so that a well-defined local normal to the chain, $\mathbf{n}(s)$, characterizes each subunit ( $s$ is the linear 

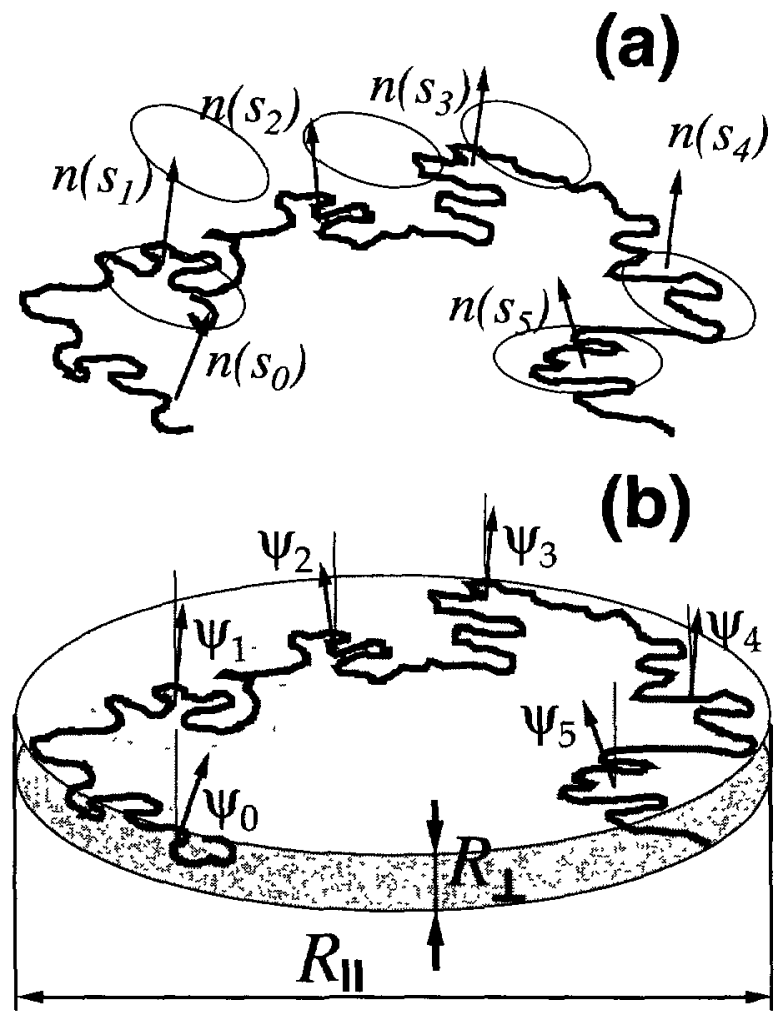

Fig. 2. - Typical conformation of a polymer with planar stiffness. The orientation of the PMFs is characterized (a) by the normal vectors, $\mathbf{n}\left(s_{2}\right)$ or (b) by deviation angles $\psi\left(s_{\imath}\right)$. Short fragments are crumbly discs with the radius $R_{\|}$and the thickness $R_{\perp}$.

coordinate measured along the chain contour). The orientation of the normal vector $\mathbf{n}(s)$ varies slowly and randomly with $s$ (see Fig. 2 a).

Let us first assume that the chain is ideal and that there are no excluded volume interactions, i.e. that distant parts of the chain are transparent to each other. As the rigidity is a local property, the various subunits of size $\lambda$ are statistically independent. As a consequence, the correlations of the normal vectors decay exponentially for $s>l$

$$
\langle\mathbf{n}(0) \mathbf{n}(s)\rangle=\exp \left(-s / l_{2}\right)
$$

It is clear that for $s \gg l$ both twisting and bending perpendicularly to the PMF affect the second persistence length, $l_{2}$, in a similar manner since for $s \gg l$ the chain has already lost the memory of its initial orientation $\mathbf{t}(0)$ in the PMF.

The persistence length $l_{2}$ in equation (2.4) measures the second rigidity of the chain with "two-dimensional" flexibility. The angle $\psi$ between $\mathbf{n}(0)$ and $\mathbf{n}(\mathrm{s})$ obeys a diffusion law for $s \ll l_{2}$.

$$
\left\langle\psi(s)^{2}\right\rangle^{(0)} \sim s / l_{2}
$$

The index $(0)$ refers to no excluded volume interactions.

In order to consider the statistics of ladder polymers such as that shown in Figure 1, we model the chain as a cylinder of diameter $d$ with two characteristic persistence lengths $l$ and 
$l_{2}$. The chain length is measured either by its contour length $L$, or by the number of Kuhn segments of length $l, N=L / l$. Our choice of a persistent model for the first rigidity, connected with $l$, is not crucial. For most of the scaling results given below the nature of the first rigidity is not important, provided that the definition (2.1) is used. The precise mechanism of the $l$-rigidity is only crucial for nematic phases with highly oriented tangent vectors $t(s)$. The corresponding generalization of the theory to any kind of flexibility is straightforward. The persistent nature of the second rigidity $l_{2}$ is however quite important throughout this paper.

\section{Conformation of Ribbon-Like Polymers in Dilute Solutions}

We discuss here the conformations of ribbon-like chains in a very dilute good solvent, when each chain can be considered as isolated. Rather short chains $\left(L \ll l_{2}\right)$ have an asymmetric shape, and can be characterized by two different sizes $R_{\|}$and $R_{\perp}$, the end-to-end distances projections on the main plane (say, on the PMF of the middle link of the chain) and on its normal vector $\left(R_{\|} \gg R_{\perp}\right.$, see Fig. $\left.2 \mathrm{~b}\right)$. We show below that such short chain have a disc-like rather than a spherical shape.

3.1. Conformation of an Isolated Ideal Chain. -- We first determine the shape of an ideal chain, $R_{\|}^{(0)}$ and $R_{\perp}^{(0)}$, ignoring any excluded-volume interactions. The results are summarized in Figure 3 (dashed lines). For short enough chains $\left(L \ll l_{2}\right.$, and hence $\left.\psi \ll 1\right)$ the shape is asymmetric and the trajectory of the chain is nearly parallel to one plane. The chain radius in this plane. $R_{\|}^{(0)}$, is that of an ordinary ideal chain with a persistence length $l[1,9]$ :

$$
R_{\|}^{(0)} \sim\left\{\begin{array}{cll}
l N=L, & \text { if } & L \lesssim l \\
l \sqrt{N}=(L l)^{1 / 2} & \text { if } & L \gtrsim l
\end{array}\right.
$$

The perpendicular chain size, $R_{\perp}^{(0)}$, is estimated as (see Fig. 2 and Eq. (2.5)):

$$
R_{\perp}^{(0)} \sim R_{\|}^{(0)} \psi^{(0)} \sim R_{\|}^{(0)}\left(L / l_{2}\right)^{1 / 2}
$$

where $R_{\|}^{(0)}$ is determined by equation (3.1) (we assume that the plane bending angle is small, $\left.\psi^{(0)}<1\right)$

$$
R_{\perp}^{(0)} \sim\left\{\begin{array}{ccc}
L^{3 / 2} / l_{2}^{1 / 2}, & \text { if } & L \lesssim l \\
L\left(l / l_{2}\right)^{1 / 2}, & \text { if } & l \lesssim L \lesssim l_{2} \\
(L l)^{1 / 2}, & \text { if } & L \gtrsim l_{2}
\end{array}\right.
$$

When $L \gtrsim l_{2}$ the chain configuration becomes isotropic: $R_{\perp}^{(0)} \sim R_{\|}^{(0)}$. We also assume that the dimensions of the whole coil are always larger than the chain diameter $d: R_{\|} \geq d ; R_{\perp} \geq d$.

According to equation (3.3) $R_{\perp}^{(0)}$ becomes of order of $d$ when

$$
L \sim L_{3} \equiv\left\{\begin{array}{lll}
l \Omega^{1 / 3}, & \text { if } \quad \Omega<1 \\
l \Omega^{1 / 2}, & \text { if } \quad \Omega>1
\end{array}\right.
$$

where we have introduced the dimensionless rigidity ratio

$$
\Omega \equiv l_{2} d^{2} / l^{3}
$$

to characterize the second rigidity $l_{2}$. For $L \lesssim L_{3}$ the apparent thickness. $R_{\perp}$, of the coil is of order $d$. 


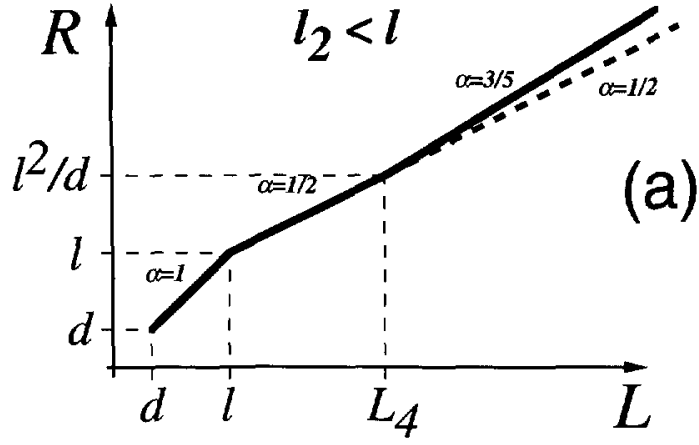

a)

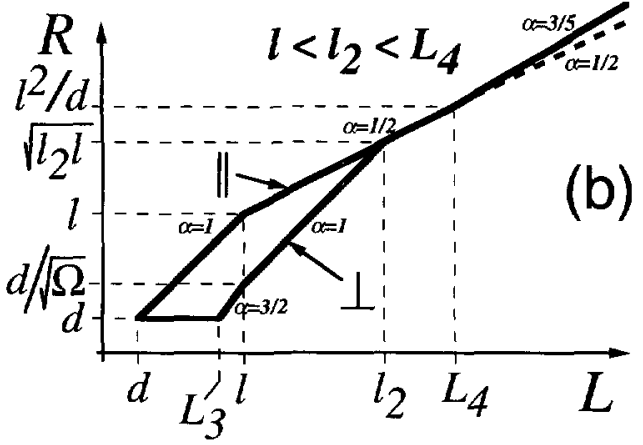

b)

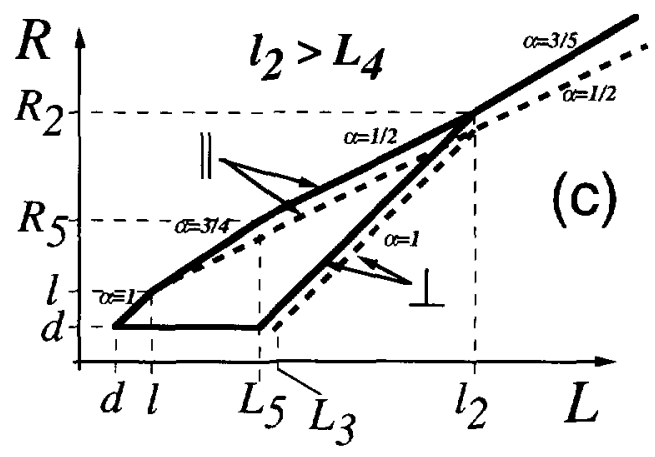

c)

Fig. 3. - Characteristic scales $R_{\|}$and $R_{\perp}$ plotted vs. contour length $L$ of a chain fragment for various values of the second rigidity $l_{2}$ : (a) $\Omega \lesssim(d / l)^{2}$; (b) $(d / l)^{2} \lesssim \Omega \lesssim 1$; (c) $\Omega \gtrsim 1 . L_{4} \equiv l^{3} / d^{2}$. The slopes $\alpha$ for the dependencies $R(L) \propto L^{\alpha}$ are shown near the corresponding lines. Self-avoiding chain (solid lines); ideal chain (dashed lines).

3.2. Conformation of an Isolated Chain in a Good Solvent. - We now use Flory-like arguments to estimate the dimensions of a non-ideal (self-avoiding) chain with two-dimensional flexibility. The Flory approach gives quite satisfactory results for the dimensions of isotropic chains in one, two and three-dimensional spaces [6]. The free energy of one chain is written as a function of the sizes $R_{\|}, R_{\perp}$ as:

$$
\mathcal{F}=2\left(\frac{R_{\|}}{R_{\|}^{(0)}}\right)^{2}+\left(\frac{R_{\perp}}{R_{\perp}^{(0)}}\right)^{2}+N n B
$$

where $n$ is the characteristic segment concentration (inside the chain) and $B$ is the second virial coefficient of two Kuhn segments of length $l$ (here and below we choose $k_{\mathrm{B}} T$ as the energy unit). The "ideal" dimensions $R_{\alpha}^{(0)}(\alpha=\|, \perp)$ have been determined in Section 3.1 (Eqs. (3.1), (3.3)). The equilibrium sizes $R_{\alpha}$ are defined by minimization of the free energy $(3.6)$, provided that these dimensions are larger than the "ideal" dimensions $R_{\alpha}^{(0)}$.

We consider here only repulsive forces. The case of attractive interactions could also be treated along the lines of reference [12] as for ordinary persistent chains.

We call the chain flat if all its links lay in "the same plane" (of thickness $d$ ). In this case we 
use "two-dimensional" values for the concentration and the virial coefficient:

$$
n=n_{2} \sim N / R_{\|}^{2} ; B=B_{2} \sim l^{2} \quad \text { for } \quad R_{\perp} \lesssim d
$$

If the chain is thicker than $d$, we call it thıck and use "three-dimensional" values for $n$ and $B$ :

$$
n=n_{3} \sim N / R_{\|}^{2} R_{\perp} ; B=B_{3} \sim l^{2} d \quad \text { for } \quad R_{\perp} \gtrsim d
$$

The values of the virial coefficient $B$ in equations (3.7), (3.8) were obtained by Onsager [13] for rods with random orientations.

The results of the minimization of the free energy (3.6) are presented in Figure 3 (solid lines). The values of the radii, $R_{\alpha}(L)$, depend strongly on the rigidity parameter $\Omega$ defined in equation (3.5).

For the case $l_{2} \lesssim l\left(\Omega \lesssim(d / l)^{2}\right)$ the chain has no PMF at any scale, i.e. the conformation of the chain is always "isotropic" (rod or coil): $R_{\|} \simeq R_{\perp} \sim R_{0}$ (see Fig. 3a) where $R_{0}$ is the radius of a persistent chain with only one rigidity $l$ ( $c f .[2])$ :

$$
R_{0} \sim\left\{\begin{array}{ccc}
L & \text { if } & d \lesssim L \lesssim l \\
(L l)^{1 / 2} & \text { if } & l \lesssim L \lesssim L_{4} \equiv l^{3} / d^{2} \\
L^{3 / 5}(d l)^{1 / 5} & \text { if } & L \gtrsim L_{4}
\end{array}\right.
$$

The cross-over length $L_{4}$ corresponds to the Fixman parameter $z\left(L_{4}\right) \equiv\left(L_{4} / l\right)^{1 / 2} B_{3} / l^{3}$ of order 1 since $B_{3} / l^{3} \simeq d / l$.

For higher rigidities $l_{2}$, when $(d / l)^{2} \lesssim \Omega \lesssim 1$ (or $l \lesssim l_{2} \lesssim l^{3} / d^{2}$ ) the various regimes are displayed in Figure 3b. The chain becomes thack at $L \sim L_{3} \equiv l \Omega^{1 / 3}$, i.e. before it reaches the cross-over from the "rod" to "semiflexible coil" regime at $L \sim l$. In this case even in the regime $L \gtrsim l_{2}$ where the chain is completely isotropic, it might be not yet swelled by the excluded volume interactions; the swelling onsets at $L \sim L_{4} \sim l(l / d)^{2} \gg l_{2}$. Thus, if $l \lesssim l_{2} \lesssim l^{3} / d^{2}$ and $L \lesssim L_{4}$, the characteristic dimensions of the coil, $R_{\|}$and $R_{\perp}$, coincide with $R_{\|}^{(0)}$ and $R_{\perp}^{(0)}$ (see Eqs. (3.1), (3.3)). On the other hand for $L \gtrsim l_{2}$, we have $R_{\|} \simeq R_{\perp} \sim R_{l_{2}=0}$ (see Eq. (3.9)), i.e. finally:

$$
\begin{array}{ccccc}
R_{\|} \sim L & \text { and } & R_{\perp} \sim d & \text { if } & d \lesssim L \lesssim L_{3} \\
R_{\|} \sim L & \text { and } & R_{\perp} \sim L^{3 / 2} / l_{2}^{1 / 2} & \text { if } & L_{3} \lesssim L \lesssim l \\
R_{\|} \sim(L l)^{1 / 2} & \text { and } & R_{\perp} \sim L\left(l / l_{2}\right)^{1 / 2} & \text { if } & l \lesssim L \lesssim l_{2} \\
R_{\|} \sim(L l)^{1 / 2} & \text { and } & R_{\perp} \sim R_{\|} & \text {if } & l_{2} \lesssim L \lesssim L_{4} \\
R_{\|} \sim\left(L^{3} l d\right)^{1 / 5} & \text { and } & R_{\perp} \simeq R_{\|} & \text {if } & L_{4} \lesssim L
\end{array}
$$

If the second rigidity is even higher $\left(l_{2} \gtrsim l^{3} / d^{2}\right.$ or $\left.\Omega \gtrsim 1\right)$ the chain passes from the regime of "flat rod" (for $L<l$ ) to that of "flat swollen coil" (for $l<L<L_{5} \equiv l \Omega^{2 / 5}$ ), then to that of "thick disc" (for $L_{5}<L<l_{2}$ ) and finally to the regime of "three-dimensional swollen coil" (for $L>l_{2}$ ) (see Fig. 3c):

$$
\begin{array}{ccccc}
R_{\|} \sim L & \text { and } & R_{\perp} \sim d & \text { if } & d \lesssim L \lesssim l \\
R_{\|} \sim l(L / l)^{3 / 4} & \text { and } & R_{\perp} \sim d & \text { if } & l \lesssim L \lesssim L_{5} \equiv l \Omega^{2 / 5} \\
R_{\| \sim(L l)^{1 / 2} \Omega^{1 / 10}} & \text { and } & R_{\perp} \sim(L d / l) \Omega^{-2 / 5} & \text { if } & L_{5} \lesssim L \lesssim l_{2} \\
R_{\|} \sim\left(L^{3} l d\right)^{1 / 5} & \text { and } & R_{\perp} \simeq R_{\|} & \text {if } & l_{2} \lesssim L
\end{array}
$$


It is worthwhile to stress here that the results of equation (3.11) are obtained by direct minimization of the free energy (3.6). An interesting feature in the "thick disc" regime is that the angular statistics of the PMF are not disturbed by excluded volume interactions. Indeed, the characteristic angles between the PMFs inside the chain can be estimated as

$$
\psi(L) \sim R_{\perp} / R_{\|}
$$

and it is clear from the comparison of equations (2.5), (3.11), (3.12) that $\psi \sim \psi^{(0)}$ This is due to the fact that the Gaussian angular statistics of (2.5) leads to a completely stretched configuration in the perpendicular direction: $R_{\perp} \propto L$ (see the second line in Eq. (3.3) and the third line in Eq. (3.11), and thus it cannot be changed by the excluded volume interactions.

3.3. Scaling Picture for the Structure of Ribbon Chains with $\Omega>1$. - The chain radii given by equation (3.11) can be interpreted in terms of "blobs". Let us divide the chain into fragments each one consisting of $N_{5} \equiv L_{5} / l \equiv \Omega^{2 / 5}$ links. The fragments ("coins") are flat and their dimensions are $R_{\|} \sim R_{5}$,

$$
R_{5} \simeq l N_{5}^{3 / 4} \sim l \Omega^{3 / 10}
$$

and $R_{\perp} \sim d$. When $L \gtrsim L_{5} \equiv N_{5} l$ a chain forms a "monisto", a kind of necklace of coins. Being flat, the fragments are strongly swollen in two dimensions, hence neighboring "coins" strongly repel each other as they are located almost in a common plane. Thus, the next "coin" in the "monisto" prefers to occupy the neighboring plane (almost parallel to the previous one but shifted by distance $d$ ), and the "monisto" as a whole occupies a sequence of almost parallel planes (see Fig. 4a). Each coin in the monisto occupies a plane in the sequence, next to the plane occupying by the previous coin. The monisto is thus strongly stretched along the axis perpendicular to these planes. The stretching is due to the strong repulsion between the coins. The thickness of such a rouleau of planes is of order $d\left(L / L_{5}\right) \sim\left(d L / l \Omega^{2 / 5}\right)$ in agreement with the result (3.11) for $R_{\perp}$ in the "thick disc" regime $\left(L_{5} \lesssim L \lesssim l_{2}\right)$. On the other hand, as soon as each "coin" occupies its own plane, the coins virtually do not meet each other and hence the statistics of the chain of "coins" parallel to these planes is Gaussian: $R_{\|} \sim R_{5}\left(L / L_{5}\right)^{1 / 2} \sim$ $(L l)^{1 / 2} \Omega^{1 / 10}$ (see Eq. (3.11). When $L$ approaches $l_{2}$, the typical angle between the "coins" is of order unity, and the picture of parallel planes fails on larger scales: instead, we get a ball-like configuration; each ball is made of "parallel" planes and has a size

$$
R_{\perp} \sim R_{\|} \sim R_{2} \equiv\left(l d l_{2}^{3}\right)^{1 / 5}
$$

At larger scales $\left(L \gtrsim l_{2}\right)$ the chain can be considered as a sequence of strongly repelling balls (see Fig. 4b). The second virial coefficient between the balls is of the order of their volume $R_{2}^{3}$. The radius of the chain is therefore $R \sim R_{2}\left(L / l_{2}\right)^{3 / 5}$ in agreement with the last regime of equation (3.11).

\section{Liquid Crystalline Phases in Semidilute Solutions of Ribbon-Like Polymers}

In the previous section we have studied the behavior of an isolated chain with planar rigidity. The next natural step is the investigation of more concentrated solutions. However, the problem is complicated by several possible orientational transitions. These transitions are related to the coil shape asymmetry of the ribbon chains (see Figs. 2, 3). Here we try to describe the various ordered phases which may appear in more concentrated solutions. These include various types of nematic as well as smectic phases. In Section 4.1 we discuss possible types of nematics, and 
a)

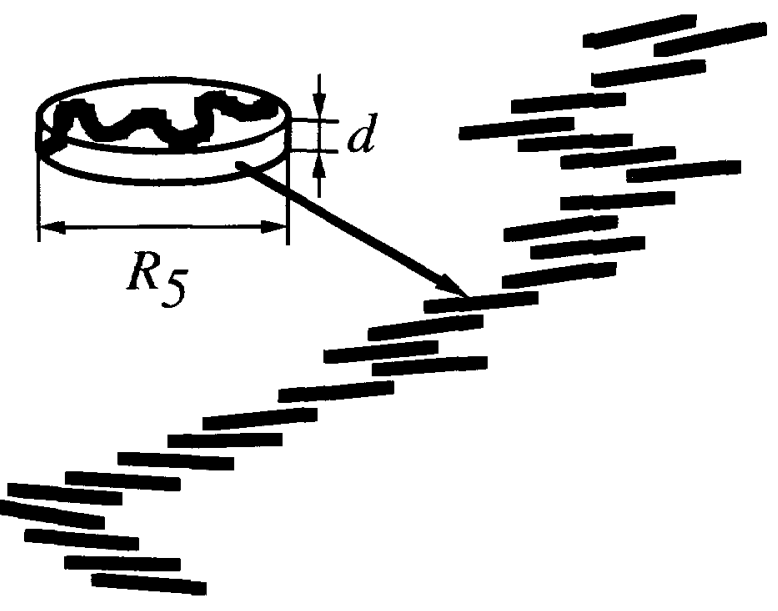

b)

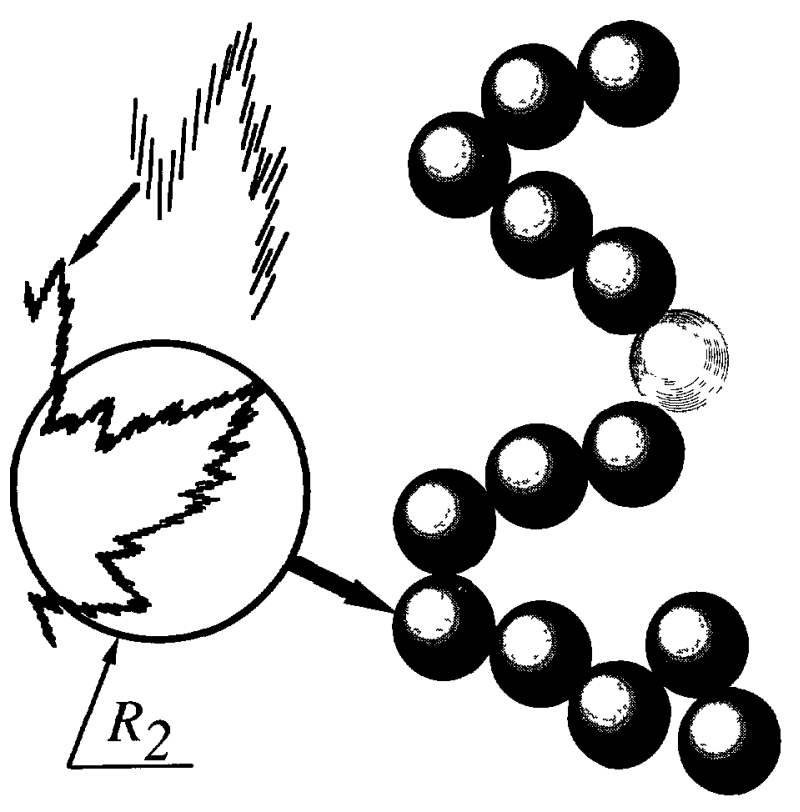

Fig. 4. - Schematic picture of a chain with high second rigidity $(\Omega \gtrsim 1)$ : a) "monisto" made of "coins" $\left.\left(d \times R_{5}^{2}\right) . L_{5} \lesssim L \lesssim l_{2} ; \mathrm{b}\right)$ three-dimensional swollen coil made of "balls" $\left(R_{2}^{3}\right), L \gtrsim l_{2}$.

in Section 4.2 more ordered liquid crystalline phases are considered. However, in the following, we only discuss the possibilities of liquid crystalline phases formation and we propose formal models for these phases. We have to postpone the discussion of the stability of these phases to the next papers of this series (Refs. $[18,19]$ ).

4.1. NEMATIC Phases, - The shape anisotropy of chains (or of fragments of chains) with two rigidities $\left(R_{\|} \gg R_{\perp}\right.$, see Figs. 3,4$)$ might induce orientational transitions. It is well-known that solutions of solid discs often form nematic phases and/or discotic phases [14-17]. Both the isotropic-nematic and nematic-discotic transitions occur when the disc concentrations is of order of $R_{\|}^{-3}$, where $R_{\|}$is the radius of the disc. However, for the ladder chains the 
corresponding "discs" are not solid, since the trajectory of the chain occupies only a small fraction of the "disc" volume (as shown for example in Figs. 2b and 4a). The orientational ordering in solutions of such "crumbly discs" (made of chains) turns out to be quite different from that of ordinary solid discs. However, we can expect that nematic ordering of crumbly discs (with discs oriented parallel to each other) is still possible under appropriate conditions. We call this phase a pancake nematic phase (or P-phase), and the ordinary isotropic phase is denoted as I-phase. As shown in Figure 5a, in the P-nematic phase the main axis of the chains, $\mathbf{t}(s)$, is oriented perpendicular to the nematic axis, whilst the normal vectors $\mathbf{n}(s)$ are oriented parallel to the nematic axis.

It is also clear that when $\Phi \gtrsim d / l$, the P-nematic solution should transform into another kind of nematic phase due to an ordering of the tangent $t$ vectors. This ordering is of the same nature as that occurring in concentrated solutions of ordinary persistent chains $[3,20-23]$, where the main axis of the chains given by the tangent vectors $\mathbf{t}(s)$, has a preferential orientation parallel to the nematic axis (this ordinary nematic phase is called here $\mathrm{N}$-phase, see Fig. 5b). However, for ribbon-like polymers $\left(l_{2} \gtrsim l\right)$ the distribution of tangent vectors $t(s)$ can be rotationally asymmetric, implying a biaxial nematic phase ("B-phase"). In the B-nematic phase both vectors $\mathbf{t}(s)$ and $\mathbf{n}(s)$ have preferential orientations in perpendicular directions, see Figure 5c.

One can characterize the order parameters in all these nematic phases by the orientation angles $\gamma$ and $\psi_{1}, \psi_{2}$ of the vectors $\mathbf{t}(s)$ and $\mathbf{n}(s)$, so that small values of $\gamma, \psi_{1}, \psi_{2}$ correspond to high order parameters. In the isotropic (I) phase we formally set the angles $\gamma=\psi_{1}=\psi_{2}=\pi / 2$ (these angles thus correspond to zero order parameters). In a highly ordered pancake-nematic P-phase, $\gamma=\pi / 2$ and $\psi_{1}=\psi_{2}=\psi \ll 1$; on the contrary $\gamma \ll 1, \psi_{1}=\pi / 2$ and $\psi_{2}=\gamma$ in the highly ordered nematic $N$-phase. Finally $\gamma \ll 1$ and $\psi_{1}, \psi_{2} \ll 1$ in the highly ordered biaxial B-phase.

The well-known arguments based on the order parameter symmetry suggest [24] that a transition between the isotropic and any orientationally-ordered phase in three dimensions must be a first order transition, while the transitions between the $\mathrm{P}$ - and $\mathrm{B}$-, or between the $\mathrm{N}$ - and B-phases could be of the second order. In the latter case the corresponding additional order parameters (associated with the angle $\psi_{1}$ for the $\mathrm{N}$-phase and the angle $\gamma$ for P-phase) change smoothly from zero (in $\mathrm{N}$ - or $\mathrm{P}$-phase) to a finite value in the $\mathrm{B}$-phase (where both orientation angles are smaller than $\pi / 2$ ).

Although a detailed analysis of nematic ordering in solutions of ribbon polymers will be presented in separate publications $[18,19]$, it is worthwhile to outline here the formal approach that we use to describe these transitions. Following [13] we characterize orientational order in the P-, N- and B-phases by trial distribution functions for the vectors $\mathbf{t}(s)$ and $\mathbf{n}(s)$. The specific form of the trial functions is not too important provided that the symmetry properties of the phases are correctly taken into account: the specific form affects only numerical coefficients in the final results $[3,23]$. Thus for the purposes of the scaling analysis which ignores numerical coefficients, we can use any smooth trial functions. We propose to use the following probability distribution functions for the orientation of the tangent vectors $\mathbf{t}(s)$ and of the normal to the PMF vectors $\mathbf{n}(s)$ :

$$
f^{\alpha} \simeq \text { const } \exp \left(-\beta_{z}^{\alpha} \cos ^{2} \theta-\beta_{y}^{a} \sin ^{2} \theta \sin ^{2} \varphi\right)
$$

where $\theta$ and $\varphi$ are the spherical coordinate angles for either $\mathrm{t}(\alpha=\mathrm{t})$ or $\mathbf{n}(\alpha=\mathbf{n})$. The prefactor of the exponential in the r.h.s. of equation (4.1) is a normalization constant. 

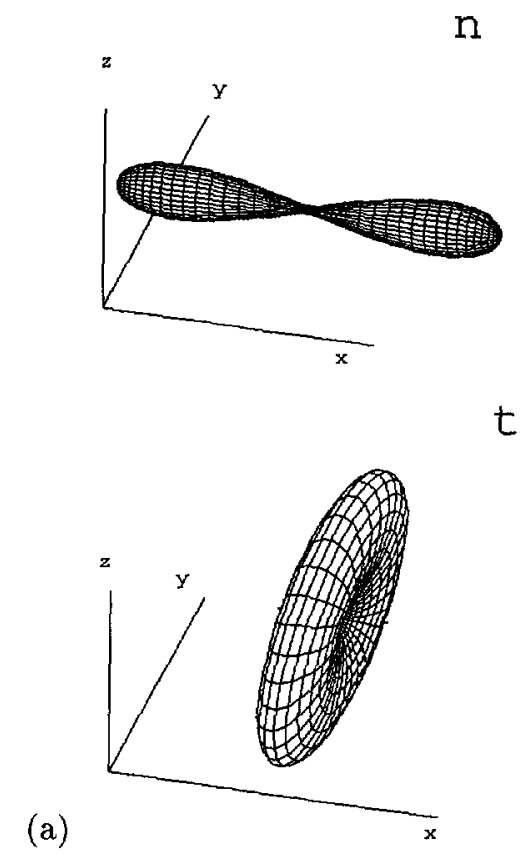

$t$

n
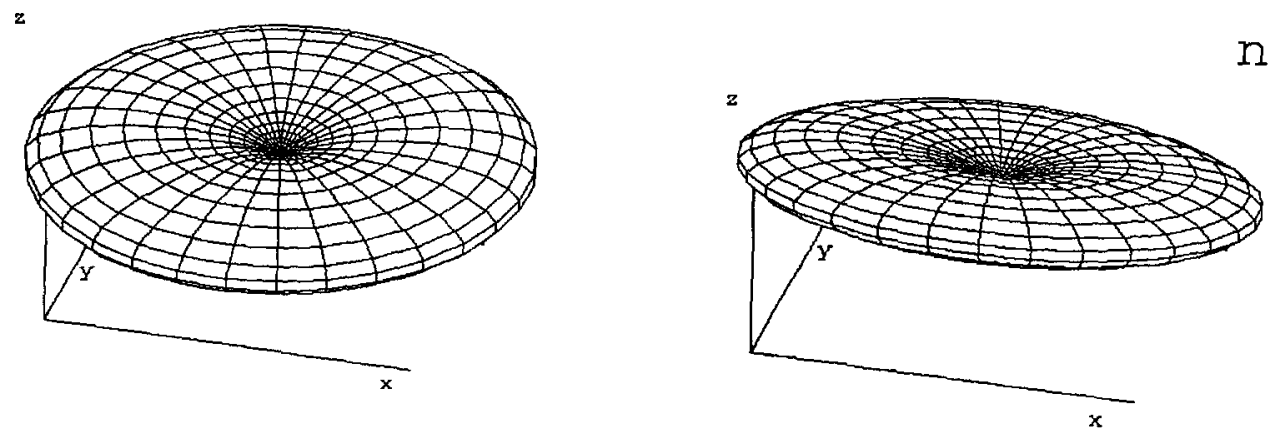

t

(b)

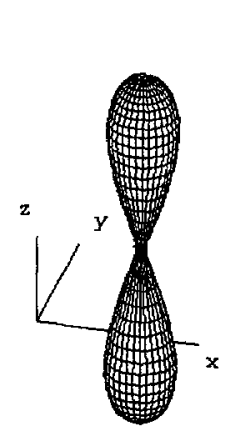

(c)

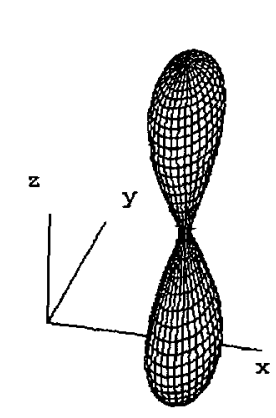

Fig. 5. - Orientation ordering in various nematic phases: orientation distributions of the tangent vectors $\mathbf{t}$ and normal vectors $\mathbf{n}$ in (a) pancake nematic phase ( $\mathrm{P}$-phase); (b) uniaxial nematic phase (N-phase); (c) biaxial nematic phase (B-phase). 
The situations illustrated in Figure 5 correspond to the following values of the parameters $\beta_{\imath}^{\alpha}$ :

$$
\begin{array}{cc}
\beta_{z}^{\mathbf{n}}=\beta_{y}^{\mathbf{n}}=-\beta_{z}^{\mathbf{t}}=-\beta_{y}^{\mathbf{t}}=\cot ^{2}(\psi), & \text { P-phase, Fig. 5a } \\
\beta_{z}^{\mathbf{n}}=-\beta_{z}^{\mathbf{t}}=\cot ^{2}(\gamma) ; \beta_{y}^{\mathbf{n}}=\beta_{y}^{\mathbf{t}}=0, & \text { N-phase, Fig. 5b } \\
\beta_{z}^{\mathbf{n}}=-\beta_{z}^{\mathbf{t}}=\cot ^{2}\left(\psi_{2}\right) ; \beta_{y}^{\mathbf{n}}=\cot ^{2}\left(\psi_{1}\right) ; \beta_{y}^{\mathbf{t}}=\cot ^{2}(\gamma)-\cot ^{2}\left(\psi_{2}\right), & \text { B-phase, Fig. 5c. }
\end{array}
$$

The distribution functions, equation (4.1), allow a study of all the nematic-like phases (I-, P-, N- or B-) simultaneously. A thermodynamic analysis of these phases can be performed according to the following program (which is actually realized in the next papers [18, 19]: As a first step we must calculate the free energy as a function of the orientation angles, $\gamma$ and $\psi_{1}$, $\psi_{2}$ (see Eq. (4.2)). The equilibrium values of the angles are then obtained by minimization of the free energy. The stable phase is the one with the lowest energy.

4.2. Smectic and Discotic (Columnar) Phases. - In smectic and discotic phases [14$17,25-28]$ the positions of the centers of masses of the particles are partially ordered in space: in the smectic phase, the particles form layers which are actually two-dimensional liquids and there is a crystalline order in one direction. The discotics are two-dimensional crystal-like structures consisting of columns, each column being a one-dimensional liquid. One can expect the formation of these more ordered liquid crystalline phases in solutions of chains with two rigidities.

The Discotic phases (D-phases) are well-known for solutions of monodisperse solid particles with disc-like symmetry (see Fig. 6c). The discotic structure implies that the discs are not only oriented (like they do in a P-nematic), but also the positions of their centers are correlated in space so that the discs form columns. The axes of the columns are parallel to the nematic axis (vertical in Fig. 6c) and form an ordered array. The period of this two-dimensional crystal is of the order of the diameter of the discs, $R_{\|}$. Inside each column the structure is liquid-like. Let us compare the P-and D-phases (see Figs. 6a and c). If the discs are ordered into columns, the phase looses translational entropy of the discs; this favors the P-phase at low concentrations. However, at concentrations near the close-packing limit, the D-phase can become more stable due to excluded volume interactions. Indeed, if one probe disc is added to the D-phase, the excluded volume interactions depend on the position of the probe: it is clear from Figure $7 \mathrm{a}$ that the free energy of the disc " 1 " is higher than that of the disc "2", as the former one interacts with the discs from two neighboring columns, and the latter one is incorporated in a single column. So as the osmotic pressure of the solution increases, the P-phase might transform into the ordered D-phase which shows a lower pressure at the same concentration.

However, from these arguments it is clear that the transition to the discotic phase is connected with the fact that the disc have a standard diameter. If the typical fluctuations of the diameter were of the order of disc diameter, there would be no difference between the discs " 1 " and " 2 " in Figure 7a, so that the driving force for a D-structure would disappear. This is actually the case for the solutions of chains with planar rigidity, which look like disc-like particles at intermediate length scale. Indeed, the characteristic dimensions of the coils, $R_{\|}$ and $R_{\perp}$ (see Sect. 3), are randomly distributed and fluctuate strongly. Hence, the D-phase is not likely to appear in these solutions.

Smectic phases (S-phases) are another possibility for solutions of solid discs (Fig. 6b). The one-dimensional crystalline structure (perpendicular to the layers) is characterized by the period of the order of the disc thickness, $R_{\perp}$. Again, the ordering transition $\mathrm{P} \rightarrow \mathrm{S}$ is connected with a loss of translational entropy and with a gain in the packing entropy which is determined by the excluded volume interactions (one can compare the free energies of the discs " 1 " and "2" in the part (b) of Fig. 7 similarly to the case considered above with Fig. 7a). The absence 


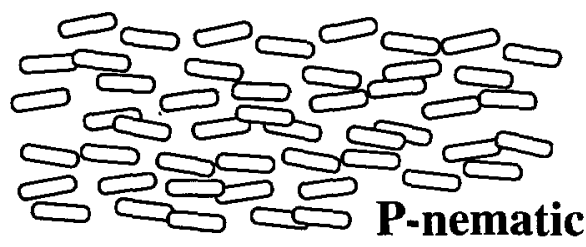

(a)

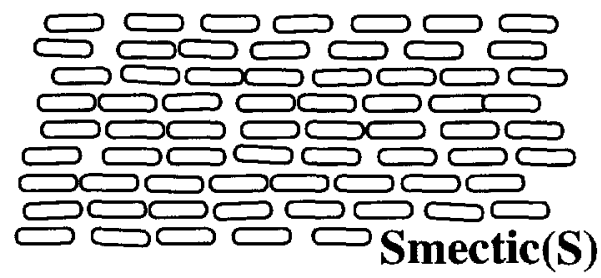

(b)

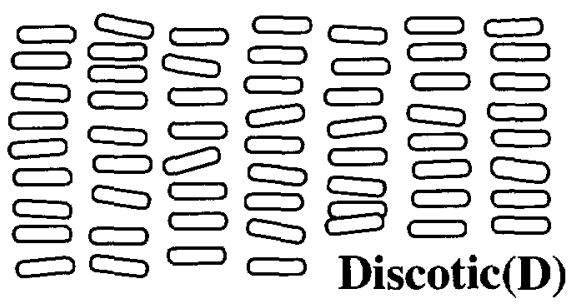

(c)

Fig. 6. - Three types of liquid-crystalline ordering in solutions of disc-like objects: (a) pancake nematic; (b) smectic; (c) discotic.
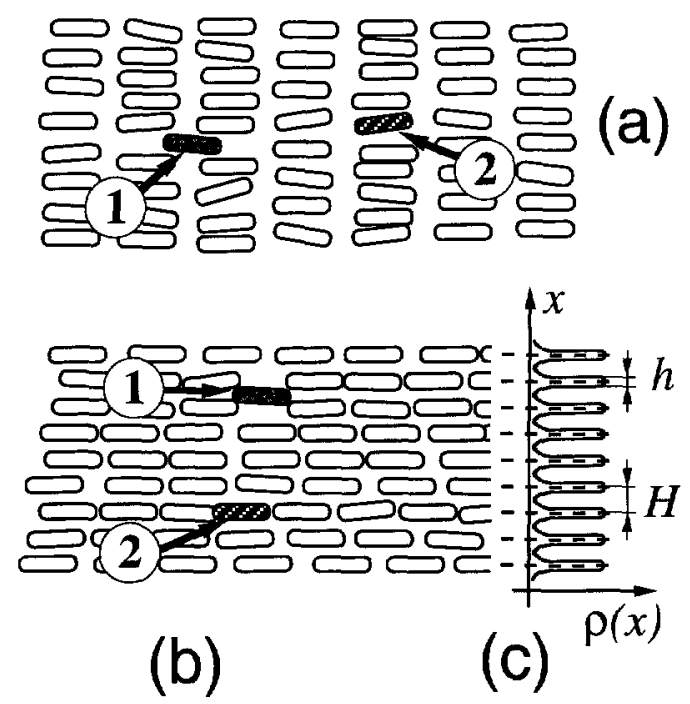

Fig. 7. - (a) Additional discs inserted into discotic phase: disc "1" interacting with two rows has a higher free energy than disc "2" inserted in a single row. (b) The same for the layered smectic structure. (c) The monomer distribution function $\rho(x)$ in a smectic phase. 
of S-phase in solutions of monodisperse solid discs is due to the competition between the $\mathrm{P} \rightarrow \mathrm{S}$ and $\mathrm{P} \rightarrow \mathrm{D}$ transitions (the discotic transition preempts the smectic transition). Smectic ordering is however known in solutions of rods (say, of length $L$ and diameter $d$ ), where smectic phases with the period $H \sim L$ were observed [17,25-28] near the close-packing limit $(\Phi \sim 1)$. In the case of chains with two rigidities, which form crumbly discs in solutions, the transition to the D-phase is suppressed and the possibility of a $\mathrm{P} \rightarrow \mathrm{S}$ transition must be considered. The stability of the S-phase is due to the fact that the chains have a standard diameter $d$, giving rise to a smectic ordering with periodicity $H \sim d$.

In the S-phases, the chains have their PMFs practically parallel to the smectic layers, so that the normal vectors to the PMFs, $n$, are strongly ordered along the axis perpendicular to the layers. This ordering of the $\mathbf{n}$-vectors is similar to that of the P-phase, and can also be described by the characteristic orientation angle, $\psi$.

In addition to the orientational order, there is also a one-dimensional crystalline order in the S-phase: the chains are arranged in layers. The chain links (monomers) are distributed inhomogeneously and periodically in space. To characterize this translational order we introduce the spatial distribution function for the points on the central axis of the chains, $\rho(x)$ (the axis $x$ is perpendicular to the layers). The function $\rho(x)$ has pronounced maximums near the centers of the layers (see Fig. 7c). The period of the function $\rho(x)$ coincides with the width of the smectic layers, $H$, the width of the peaks, $h(h \lesssim H)$, characterizes the degree of smectic ordering in the S-phase: the smaller $h$, the higher the one-dimensional crystalline order. When $h \sim H$ the S-phase transforms into a P-phase.

The width $h$ is actually the width of the inter-layer gaps (cf. parts (b) and (c) of Figure 7): for a given smectic periodicity $H$, the distribution function $\rho(x)$ is smeared for entropic reasons around its maximum until objects (discs, chains, etc.) of neighboring layers touch each other. If the S-phase is formed by objects of width $d$, the smectic periodicity is:

$$
H \simeq d+h,
$$

It is clear that $H$ typically ranges between $d$ and $2 d$. The objects strongly interfere inside the layers, but the inter-layer interactions are much weaker, because the probability density $\rho(x)$ to find the object between the layers is rather low (for $h \ll H$ ). So the total interaction energy of the S-phase is basically dependent on the intra-layer concentration. This intra-layer concentration can be determined as the two-dimensional concentration of objects inside the layers. If the volume-averaged concentration of the objects in the S-phase is $c$, the intra-layer concentration $c_{2}$ can be estimated as:

$$
c_{2} \simeq c H \simeq c d\left(1+\frac{h}{d}\right)
$$

Hence, $c_{2}$ can change by a factor of 2 in the interval of possible gap widths $h(0<h \lesssim d)$.

Now one can compare S-phases with different distribution functions $\rho(x)$. At a given concentration $c$, a decrease of the gap width $h$ causes: 1) entropy loss due to the formation of the layer structure (of order $\Delta S \sim \ln (h / H)$ for each subunit); 2 ) a decrease of the interaction free energy due to a decrease of the intra-layer concentration $c_{2}$ in accordance with equation (4.4). A competition between these two factors gives the value of the equilibrium order parameter $H / h$. We will show in the following paper [19] that for high enough rigidities $l_{2}$, the competition between these two factors leads to the formation of a smectic phase with a high order parameter $(H / h \gg 1)$.

To further illustrate this point, we consider here ribbons with an infinite second rigidity, $l_{2}=$ $\infty$, at a rather high concentration: $c \gg c^{* * *} \simeq\left(R_{\perp} R_{\|}^{2}\right)^{-1}$ where $R_{\perp} \simeq d$ and $R_{\|} \simeq\left(L^{3} l\right)^{1 / 4}$ 
are the sizes of the chain (see Eq. (3.11)). One can prove easily that these disc-like chains form a strongly ordered S-phase. Indeed, at $c \sim c^{* * *}$ the discs occupy the whole volume, this corresponds to an intra-layer concentration $c_{2}^{* * *} \sim R_{\|}^{-2}$ At a higher concentration, the chains strongly overlap and the free energy per chain in a S-phase is (we use here the standard scaling formula [6] for the interaction energy and Eqs. (4.3), (4.4)):

$$
\mathcal{F}=\left(\frac{c_{2}}{c_{2}^{* * *}}\right)^{v}-\ln \left(\frac{h}{H}\right) \simeq\left(c d\left(1+\frac{h}{d}\right) R_{\|}^{2}\right)^{v}+\ln \left(1+\frac{d}{h}\right),
$$

where $v=\frac{1}{\mathcal{D} \nu-1}=2$ (here $\mathcal{D}=2$ and $\nu=3 / 4$ ) is the positive critical exponent for the variation of the chemical potential as a function of concentration for two-dimensional polymers in a good solvent. The minimization of the free energy for $c \gg c^{* * *}$ gives the equilibrium value $h \sim d\left(c^{* * *} / c\right)^{2} \ll H \simeq d$. Thus a highly ordered S-phase is indeed stable.

In the simplest smectic phase the layers are isotropic (the structure of the phase is invariant by rotation around the $x$-axis, which is perpendicular to the layers). Such smectic phases are usually named $\mathrm{S}_{\mathrm{A}}$-smectics [14]. These $\mathrm{S}_{\mathrm{A}}$-phases can be characterized by the periodicity $H \sim d$, and also by two other parameters: $h$ (the characteristic width of the inter-layer gaps) and $\psi$ (characteristic orientation angles of the normals to the PMFs of the chains).

However, due to the local asymmetry of the chains $(d \ll l)$ when the volume fraction occupied by the chains in space, $\Phi$, exceeds $d / l$, a transition from $\mathrm{S}_{\mathrm{A}}$-phase into a more ordered phase takes place. The origin of this transition is very similar to the ordering of the tangent vectors $t$ in the $\mathrm{N}$ - and B-phases: they prefer to orient along a given axis in order to decrease the interaction energy $[3,14]$. This axis (say, axis $z$ ) is perpendicular to the axis of preferential orientation of $\mathbf{n}$-vectors ( $c f$. Sect. 4.1), and lays inside the smectic layers. This structure (which is usually called smectic- $\mathrm{C}$ [14]) does not have a rotational symmetry around the $x$-axis.

The $\mathrm{S}_{\mathrm{C}}$-phases can be characterized by the inter-layer gap width, $h$, and by the two characteristic angles, $\psi$ and $\gamma$, which are needed to describe the ordering of the $\mathbf{t}-$ and $\mathbf{n}$-vectors.

\section{Discussion and Conclusions}

In this paper we have considered the behavior of a polymer chain with anisotropic rigidity. If the chain structure has an intrinsic mirror symmetry but not a rotational symmetry, the chain might reveal an anisotropic stiffness. We have theoretically considered the case when this anisotropy is strong enough $i$.e. when the rigidities inside the mirror plane $(l)$ and outside the mirror plane $\left(l_{2}\right)$ differ significantly, $l_{2} \gtrsim l$. In this regime the chain conformation is similar to a two-dimensional walk (if the molecular weight is not too large) in a plane which we call the plane of main flexibility (PMF). The two-dimensional statistics of the chain can be characterized by a Kuhn segment $l$. For longer chains the deviations from the initial PMF become significant. The amplitude of these deviations is characterized by another persistence length $l_{2}$. In Section 2 we have formulated a model for chains with two rigidities and we have shown that the two parameters $\left(l\right.$ and $\left.l_{2}\right)$ are sufficient to describe the chain statistics, independently of the detailed microscopic nature of the deviation from the PMF which can be due to either bending outside the PMF, or twisting of the chain, or both.

In Section 3, we have investigated the behavior of a long isolated chain with anisotropic rigidity, and in Section 4 we discussed and classified the possible ordered phases which could be observed in semidilute solutions.

The behavior of chains with two rigidities depends crucially on the value of the dimensionless parameter $\Omega \equiv l_{2} d^{2} / l^{3}$. If $\Omega \lesssim 1$ then the effect of the second rigidity $l_{2}$ is relatively weak. However, for contour lengths $L \lesssim l_{2}$, the general shape of the chain is still anisotropic (disc-like). 
We expect this anisotropy to lead to the formation of nematic phases in more concentrated solutions, namely both the pancake and the biaxial nematics (phases $P$ and $B$ ), with extra ordering due to the second rigidity.

In the limit of very high $l_{2}(\Omega \gtrsim 1)$ the effect of the second rigidity is more pronounced. For the single chain behavior we have found a tremendous shift of the characteristic scales: the classical three-dimensional swollen behavior exists only for $L \gtrsim l_{2} \gg L_{4} \equiv l^{3} / d^{2}$. At rather short scales an ordinary two-dimensional behavior is expected in the flat rod regime (for $L \lesssim l$ ) and the flat swollen coil regime (for $l \lesssim L \lesssim L_{5}$, Eq. (3.11)). At intermediate length scale $\left(L_{5} \lesssim L \lesssim L_{4}\right)$ a regime of "thick discs" is found, implying quasi-Gaussian statistics parallel to the PMF and highly stretched statistics perpendicular to the PMF. We have also constructed a scaling blob picture to describe the conformation of isolated chains with $\Omega \gtrsim 1$. In the high second rigidity regime we expect the formation of not only pancake $(P)$ nematic phases, but also of smectic phases (of types $\mathrm{A}$ and $\mathrm{C}$ ). The smectic phases can appear if the coils are flat, so that the chains have a standard thickness $(d)$ in one direction $(x)$, but are highly fluctuating coils in the two other directions (along the $y z$ plane, parallel to the PMF).

It should be stressed that rather short chains $\left(L \ll l_{2}\right)$ always have an asymmetric disclike shape and can be characterized by two different sizes $R_{\|}$and $R_{\perp}$ if the chain rigidity is anisotropic $\left(l_{2}>l\right)$. The fact that the chain has a disc-like rather than spherical shape could be probed experimentally using sedimentation, viscosimetry or radiation scattering techniques (see, e.g. the corresponding chapters in [29]). This asymmetry might be very important in strongly non-linear regimes (high-rate sedimentation, etc.) or when additional ordering of the chains is forced by an external field. We hope that our results will inspire experiments in these directions.

Among the systems which can be good examples of polymers with two different rigidities, we would like to mention first ladder polymers (see Fig. 1), where the chain structure indeed implies a two-dimensional random walk statistics. Normally these polymers are characterized by a low first rigidity (in fact for the polymer shown in Fig. 1a $l \sim d$ ), and by a high second rigidity: $l_{2} \gtrsim 100 l$ (these estimations are based in a figure shown in reference [30] which illustrates the conformation of a ladder polymer obtained in all-atom molecular dynamics simulations). As far as we know, the experimental study of the solutions of this family of ladder polymers is still in the very early stage (even the experimental data on isolated coils sizes are not available yet). However, it is clear from the theory presented here that this system corresponds to the case of extremely high second rigidity, $\Omega \gtrsim 1$, thus the results shown in Figure $3 \mathrm{c}$ are appropriate for this system.

Another system, which should show a behavior close to that described here, is a selfassembling (or so-call "living") "ribbon polymer" made of rod-like fragments which strongly attract each other by their opposite sides [31]. The elementary units of these polymers are peptide rod-like fragments (of length $\sim 80 \AA$ and diameter $\sim 10 \AA$ ) which have about $m \simeq 20 \div 30$ pairs of attracting points located on the rod surface along two lines parallel to the axis and diametrically opposite; these rods form a kind of $\beta$-sheet (or barrel, like in ordinary proteins) in the form of a ribbon stabilized by hydrogen bonds between attracting points (see the sketch of Fig. 8). The length of the ribbon can be extremely large (as the association energy between rods is about $30 k_{\mathrm{B}} T$ ), and electron micrographs prove that the ribbons are practically defectless [31].

From the point of view of our theoretical approach this system can be treated as follows. For the excluded volume interactions in solutions only the larger size in the chain cross-section is important, so that $d \simeq 80 \AA$. The persistence lengths can be estimated as

$$
l \simeq a / \Delta \gamma^{2} \text { and } l_{2} \simeq a / \Delta \psi^{2}
$$




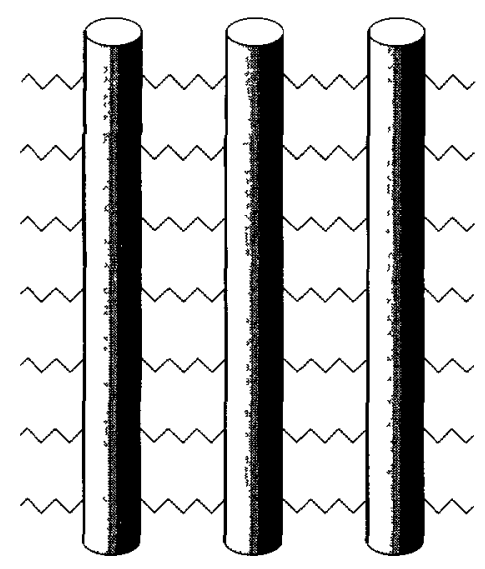

a)

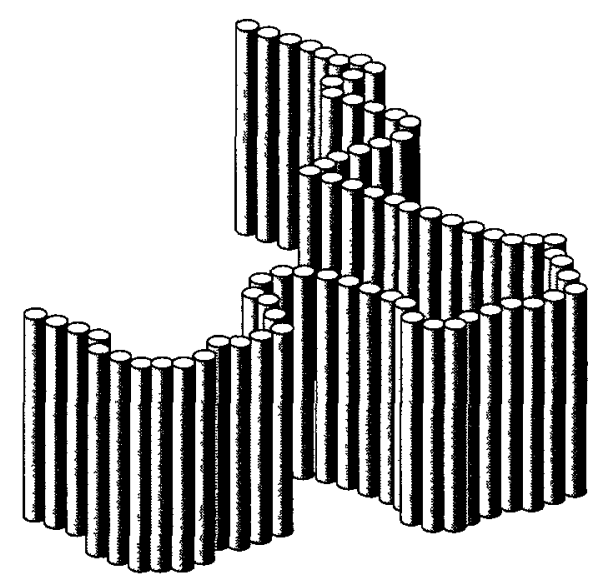

b)

Fig. 8. - A living ribbon polymer formed by peptide rod-like fragments: a) local structure: the rods attract each other by the opposite sides; b) a self-assembling ribbon at larger scales.

where $a$ is the link length (the rod diameter, $\sim 10 \AA$ ), and $\Delta \gamma$ and $\Delta \psi$ are the typical orientation deviations between neighboring rods. It is clear that as the axial ratio of the rods is high enough $(\sim 80: 10)$, the ratio of $l_{2} / l$ is also high. Indeed, if the attraction potentials were spring-like (with rigidity $\kappa$ ), and the rods had square cross-sections, the typical angles would be: $\Delta \gamma^{2} \sim\left(m \kappa a^{2}\right)^{-1}$ and $\Delta \psi^{2} \sim\left(m \kappa d^{2}\right)^{-1}$ (where $m$ is the number of springs between neighboring rods, and as everywhere in this paper $\left.k_{\mathrm{B}} T=1\right)$, hence the ratio $l_{2} / l \sim(d / a)^{2} \sim 50$. For real hydrogen short-range potentials and non-square cross-sections of the rods this ratio is even higher.

The peptide systems thus provide another example of a polymer-like object with highly anisotropic rigidity $l_{2} \gg l$ (the absolute values of the rigidities are determined by the parameter $\kappa$ and can vary depending on the solvent in which the living polymer is formed). In principle, with shorter rods (smaller $m$ ) one can get a system with closer values of $l_{2}$ and $l$. It is wellknown [32], that the apparent size $L$ is also concentration-dependent, $L \propto \Phi^{1 / 2}$, which should be taken into consideration in the analysis of the "coil shapes" for the self-assembling tape system of reference [31]. Unfortunately, systematic experimental data on the self-assembling ribbons are not available yet. To our knowledge reference [31] is the only work dealing with these systems.

The next step in the theoretical treatment of polymers with anisotropic rigidities, a detailed analysis of the nematic and smectic ordered phases and the construction of the complete diagram of states, is possible within the framework of the liquid crystalline models outlined in Section 4, using a scaling analysis similar to that of semidilute solutions [6]. This is the subject of the following publications of this series $[18,19]$.

\section{Acknowledgments}

The authors are very grateful for valuable discussions to Profs. A.-D. Schlüter, N. Boden, A.Yu. Grosberg, T.C.B. McLeish. This work was supported by Russian Fundamental Research Foundation (Russia) and CNRS (France). 


\section{References}

[1] Grosberg A.Yu. and Khokhlov A.R., Statistical physics of macromolecules, Engl. transl. (AIP, NY, 1994).

[2] Schaefer D.W., Joanny J.F. and Pincus P., Macromolecules 13 (1980) 1280.

[3] Semenov A.N. and Khokhlov A.R., Sov. Phys. Usp. 31 (1988) 988.

[4] Odijk T., Macromolecules 19 (1986) 2313.

[5] Vroege G.J. and Lekkerkerker H.N.W., Rep. Prog. Phys. 55 (1992) 1241.

[6] de Gennes P.G., Scaling Concepts in Polymer Physics (Cornell Univ. Press, Ithaca, NY, 1978).

[7] Vogel T., Blatter K. and Schlüter A.-D., Makromol. Chem., Rapid Commun. 10 (1989) 427.

[8] Schlüter A.-D., Adv. Mater. 3 (1991) 282.

[9] Flory P.J., Principles of Polymer Chemistry (Cornell University Press, Ithaca and New York, 1953).

[10] Kratky O. and Porod G., Rec. Trav. Chim. 68 (1949) 1106.

[11] Flory .P.J., Statistical Mechanics of Chain Molecules (Interscience, New York, 1969).

[12] Birshtein T.M. and Pryamitsyn V.A., Polym. Sci. USSR 29 (1987) 2039; Macromolecules 24 (1991) 1554.

[13] Onsager L., Ann. N.Y. Acad. Sci. 51 (1949) 627.

[14] de Gennes P.G. and Prost J., The Physics of Liquid Crystals (Claredon Press, Oxford, 1993).

[15] Somoza A.M. and Tarazona P., Phys. Rev. Lett. 11 (1988) 2566.

[16] Taylor M.P., Hentschke R. and HerzfeldJ., Phys. Rev. Lett. 62 (1989) 800; 1577; Phys. Rev. A 40 (1989) 1678.

[17] Stroobants A., Lekkerkerker H.N.W. and Frenkel D., Phys. Rev. Lett. 57 (1986) 1452; Phys. Rev. A 36 (1987) 2929.

[18] Nyrkova I.A., Semenov A.N. and Joanny J.F., Part II of the present paper series.

[19] Nyrkova I.A., Semenov A.N. and Joanny J.F., Part III of the present paper series.

[20] Flory P.J., Abe A. and Frost R.S., Macromolecules 11 (1978) 1119; 1122; 1126.

[21] Flory P.J., Macromolecules 11 (1978) 1138; 1141.

[22] Khokhlov A.R. and Semenov A.N., Physica A 108 (1981) 546.

[23] Khokhlov A.R. and Semenov A.N., J. Stat. Phys. 38 (1985) 161.

[24] Landau L.D. and Lifshitz E.M, Statistical Physics, 3rd ed., Part 1, (Pergamon Press, Oxford, 1980).

[25] Frenkel D., Lekkerkerker H.N.W. and Stroobants A., Nature 332 (1988) 822.

[26] Poniewierski A. and Holyst R., Phys. Rev. Lett. 61 (1988) 2461.

[27] Somoza A.M. and Tarazona P., J. Chem. Phys. 91 (1989) 517.

[28] Hasino M., Nakano H. and Kimura H., J. Phys. Soc. Jpn 46 (1979) 1709.

[29] Tzvetkov V.N., Eskin V.E. and Frenkel S.Ya., Structure of macromolecules in solution, (Nauka, M., 1964).

[30] Schürmann B.L., Enkelmann V., Löffler M. and Schlüter A.-D., Angew. Chem., Int. Ed. Engl. 32 (1993) 123.

[31] Aggeli A., Boden N., Findlay J.B.C., Knowles P.F. and McLeish T.C.B., Self-Assembly of Nanotapes based on Oligomeric Peptides, submitted to Nature.

[32] Israelachvili J.N., Intermolecular and Surface Forces (Academic Press, NY, 1985). 